\section{CPS-290 BORTEZOMIB INDUCED ACUTE NECROHAEMORRHAGIC PANCREATITIS: CASE REPORT}

L Martinez-Dueñas*. Hospital San Agustin, Pharmacy, Jaen, Spain

10.1136/ejhpharm-2021-eahpconf.122

Background and importance Bortezomib is a proteasome inhibitor indicated for the treatment of multiple myeloma. Its most common adverse effects include haematological, gastrointestinal and peripheral neuropathy. Pancreatitis is a rare serious adverse effect.

Aim and objectives We describe the case of a patient with newly diagnosed multiple myeloma who developed pancreatitis during treatment with bortezomib.

Material and methods A 64-year-old male patient started treatment with bortezomib $1.3 \mathrm{mg} / \mathrm{m}^{2}$ and lenalidomide $15 \mathrm{mg}$. When he went to the emergency room, he had received three doses of bortezomib. He reported abdominal pain, nausea and intolerance to ingestion. Feverish, exploration and complementary tests: BP 115/67 mm Hg, HR 65 bpm, SATO2 93\% baseline, 110000 platelets, 510 lymphocytes, INR 3.5, glucose 105, urea 130, Cr 1.76, uric 8.7, FG 41, lipase 2234, amylase 606, $\mathrm{Na} 130, \mathrm{~K} 5.5$, PCR 60.9.

Results The patient was admitted to internal medicine with haematology support. Treatment for multiple myeloma (bortezomib, dexamethasone, lenalidomide) was suspended and antibiotic and supportive treatment was started. Progressive worsening, evolving to multiorgan failure, occurred. Abdominal CT was performed, showing acute pancreatitis and Balthazar score; despite very high mortality by itself, the risk is higher due to multiple myeloma. The patient was admitted to the intensive care unit on the second day (tachypnoea 25 rpm, nasal gasses at $3 \mathrm{bpm}, \mathrm{O}_{2}$ sat 93\%, BP 70/50, Fc 90 bpm, platelets 41000 , leukos 4170, neutroph 90\%, urea 197, creatinine 4.81, $\mathrm{Na}$ 131, K 6.6, CRP 89, procalcitonin 367, prothrombin activator 40\%, APTT 54, fibrinogen 889 .

The diagnosis was based on the exclusion of other common causes of pancreatitis, according to the orange tree algorithm. Elevation of pancreatic enzymes occurred within a few days of the administration of bortezomib. The patient remained sedated and analgesic, endotracheal intubation, anuria, hyperlactacidaemia with a fever of $40^{\circ} \mathrm{C}$ despite paracetamol, metamizole and physical media. The patient was in a state of multiorgan failure, refractory to all measures, causing death on the third day of admission.

Conclusion and relevance The mechanism by which pancreatitis occurs is unclear. It could be related to drug toxicity, or allergic or immune mediated reactions. Although pancreatitis secondary to bortezomib is considered a rare side effect, physicians should be aware that it can occur in patients receiving this treatment.

\section{REFERENCES AND/OR ACKNOWLEDGEMENTS}

Conflict of interest No conflict of interest

\section{CPS-291 PALBOCICLIB: EARLY NEUTROPENIA AS A PHARMACODYNAMIC MARKER IN A REAL WORLD SETTING?}

\footnotetext{
${ }^{1} X$ Fernández-Sala, ${ }^{1} M E$ Navarrete-Rouco, ${ }^{1} \mathrm{~L}$ Río-No*, ${ }^{2} \mathrm{~J}$ Albanell, ${ }^{1} \mathrm{D}$ Conde-Estévez. ${ }^{1}$ Hospital Del Mar, Pharmacy Department, Barcelona, Spain; ${ }^{2}$ Hospital Del Mar, Oncology Department, Barcelona, Spain
}

Background and importance The most frequent adverse effect of palbociclib is neutropenia, resulting in dose reductions and treatment interruptions. Recently, it has been reported that early palbociclib related neutropenia was associated with a prolonged progression free survival (PFS) ${ }^{1}$. However, there are no analysis data based on the real world setting, outside of clinical trials.

Aim and objectives To determine whether early neutropenia in our cohort of patients was associated with disease response to palbociclib combined with fulvestrant or an aromatase inhibitor.

Material and methods This was a retrospective study including all patients who started treatment with palbociclib between December 2016 and January 2020. Demographic and clinical data were obtained from the electronic clinical records. Primary endpoints included both PFS and overall survival (OS). Early neutropenia was defined as the nadir absolute neutrophil count (ANC) during the first two cycles of treatment. PFS and OS were analysed with Kaplan-Meier survival curves comparing neutropenia grades using the log rank test to check differences between survival curves. Multivariate Cox proportional hazard regression model was also used to predict OS.

Results A total of 61 patients were included. Demographic and clinical characteristics are shown in table 1 .

28 patients (45.9\%) stopped treatment and 24 (85.7\%) discontinued due to progression. 25 patients $(41.0 \%)$ required $\geq 1$ dose reduction. In the first two cycles, 54 patients $(88.5 \%)$ experienced grade 1-4 neutropenia. Patients who experienced grade 2-4 neutropenia in the first two cycles were associated with significantly prolonged median OS (log rank $\mathrm{p}=0.019$ ). However, there was no significant association with prolonged median PFS ( $\log$ rank $\mathrm{p}=0.572)$. After adjusting for potential cofounders (baseline ACN, age and weight), grade 2-4 neutropenia remained significantly and independently associated with prolonged OS (HR 0.26, 95\% CI 0.09 to $0.77, \mathrm{p}=0.015$ ).

\begin{tabular}{ll} 
Abstract 4CPS-291 Table 1 & \\
\hline & Total patients $(\mathrm{n}=61)$ \\
\hline Age (years) (mean \pm SD) & $61 \pm 11.8$ \\
Women ( $(\%))$ & $60(98.4)$ \\
Weight $(\mathrm{kg})($ mean \pm SD) & $65.8 \pm 14.5$ \\
Baseline ECOG PS 0-1 (n (\%)) & $56(91.8)$ \\
Line of therapy (n (\%)) & \\
1 & $41(67.2)$ \\
2 & $14(23.0)$ \\
$\geq 3$ & $6(9.8)$ \\
Concomitant drug (n (\%)) & $25(41.7)$ \\
Fulvestrant & $36(59.0)$ \\
Aromatase inhibitor & $5.0 \pm 2.6$ \\
Baseline ANC (mean $\pm S D)$ & \\
\hline
\end{tabular}

Conclusion and relevance Early neutropenia was significantly associated with a prolonged OS, supporting the suggestion that neutropenia could be a pharmacodynamic marker for palbociclib dosing.

\section{REFERENCES AND/OR ACKNOWLEDGEMENTS}

1. McAndrew NP, et al. Br J Cancer 2020;123:912-18.

Conflict of interest No conflict of interest 